EUROPEAN JOURNAL OF PURE AND APPLIED MATHEMATICS

Vol. 13, No. 3, 2020, 587-607

ISSN 1307-5543 - www.ejpam.com

Published by New York Business Global

\title{
A Unification of the Generalized Multiparameter Apostol-type Bernoulli, Euler, Fubini, and Genocchi Polynomials of Higher Order
}

\author{
Nestor G. Acala \\ Mathematics Department, College of Natural Sciences and Mathematics, \\ Mindanao State University, Marawi City, Lanao Del Sur, Philippines
}

\begin{abstract}
Most unifications of the classical or generalized Bernoulli, Euler, and Genocchi polynomials involve unifying any two or all of the three special types of polynomials (see, $[1,4,9,18,19$, $21,24-26,30,31]$ ). In this paper, we introduce a new class of multiparameter Fubini-type generalized polynomials that unifies four families of higher order generalized Apostol-type polynomials such as the Apostol-Bernoulli, Apostol-Euler, Apostol-Genocchi, and Apostol-Fubini polynomials. Moreover, we obtain an explicit formula of these unified generalized polynomials in terms of the Gaussian hypergeometric function, and establish several symmetry identities.
\end{abstract}

2020 Mathematics Subject Classifications: 11B68, 11B73, 33C05, 05A10, 11B83

Key Words and Phrases: Fubini polynomials, Bernoulli polynomials, Euler polynomials, Genocchi polynomials, Apostol-type polynomials, Ordered Bell polynomials, Gauss hypergeometric function, Generalized Bernoulli polynomials, Generalized Euler polynomials, Generalized Genocchi polynomials, Generalized Fubini polynomials

\section{Introduction}

In recent years, extensive researches on various families of numbers and polynomials such as the Bernoulli numbers and polynomials, Euler numbers and polynomials, Genocchi numbers and polynomials, Fubini numbers and polynomials, and also their generalizations and unifications (see, for instance the recent works of $[1,3,4,10,11,17,25,26,28,31]$ ) have become popular due to the abundance of their applications in many branches of mathematics such as in $p$-adic analytic number theory, umbral calculus, special functions and mathematical analysis, numerical analysis, combinatorics and other related fields. This motivates the author to obtain and explore a new unification of some of the recent generalizations of these special types of polynomials.

In this section, we present some of the known generalizations of Bernoulli, Euler, Genocchi, and Fubini polynomials of higher order. Throughout this paper, we use the

DOI: https://doi.org/10.29020/nybg.ejpam.v13i3.3757

Email address: nestor.acala@gmail.com (N. G. Acala) 
usual notations $\mathbb{N}, \mathbb{Z}, \mathbb{R}$, and $\mathbb{C}$ for the sets of natural numbers, integers, real numbers, and complex numbers respectively. Also, we let $\mathbb{N}_{0}:=\mathbb{N} \cup\{0\}, \mathbb{Z}^{-}:=\{-1,-2,-3, \cdots\}$, and $\mathbb{Z}_{0}^{-}:=\mathbb{Z}^{-} \cup\{0\}$.

The bivariate Fubini polynomials of order $\alpha$ is defined through the generating function

$$
\frac{e^{x t}}{\left[1-y\left(e^{t}-1\right)\right]^{\alpha}}=\sum_{n=0}^{\infty} F_{n}^{(\alpha)}(x, y) \frac{t^{n}}{n !}(\operatorname{see}[12,13,15,17]) .
$$

When $\alpha=1, F_{n}^{(1)}(x, y):=F_{n}(x, y)$, the two-variable Fubini polynomials given by

$$
\frac{e^{x t}}{1-y\left(e^{t}-1\right)}=\sum_{n=0}^{\infty} F_{n}(x, y) \frac{t^{n}}{n !}(\text { see }[10,11,16]) .
$$

Moreover, setting $x=0$ in (1), we obtain

$$
F_{n}^{\alpha}(0, y):=F_{n}^{(\alpha)}(y) \text { and } F_{n}^{(\alpha)}(1):=F_{n}^{(\alpha)}
$$

where $F_{n}^{(\alpha)}(y)$ and $F_{n}^{(\alpha)}$ are called the higher order Fubini polynomials and the higher order Fubini numbers respectively (see $[6,14])$. For $\alpha=1$

$$
F_{n}^{(1)}(0, y):=F_{n}(y) \text { and } F_{n}^{(1)}(1):=F_{n},
$$

where $F_{n}(y)$ are the classical Fubini polynomials or the ordered Bell polynomials, and $F_{n}$ are the classical Fubini numbers or the ordered Bell numbers (see $[2,27]$ ).

The classical Bernoulli polynomials $B_{n}(x)$, Euler polynomials $E_{n}(x)$, and Genocchi polynomials $G_{n}(x)$ together with their natural higher order generalizations $B_{n}^{(\alpha}(x), E_{n}^{(\alpha}(x)$, and $G_{n}^{(\alpha}(x)$ are usually defined by means of the generating functions (see [1, 3, 4, 29])

$$
\begin{aligned}
& \left(\frac{t}{e^{t}-1}\right)^{\alpha} e^{x t}=\sum_{n=0}^{\infty} B_{n}^{(\alpha)}(x) \frac{t^{n}}{n !} \quad(|t|<2 \pi, \alpha \in \mathbb{C}), \\
& \left(\frac{2}{e^{t}+1}\right)^{\alpha} e^{x t}=\sum_{n=0}^{\infty} E_{n}^{(\alpha)}(x) \frac{t^{n}}{n !} \quad(|t|<\pi, \alpha \in \mathbb{C}), \\
& \left(\frac{2 t}{e^{t}+1}\right)^{\alpha} e^{x t}=\sum_{n=0}^{\infty} G_{n}^{(\alpha)}(x) \frac{t^{n}}{n !} \quad(|t|<\pi, \alpha \in \mathbb{C}) .
\end{aligned}
$$

Hence,

$$
B_{n}^{(1)}(x):=B_{n}(x) \quad E_{n}^{(1)}(x):=E_{n}(x) \quad \text { and } \quad G_{n}^{(1)}(x):=G_{n}(x) .
$$

The classical Bernoulli numbers $B_{n}$, Euler numbers $E_{n}$, and Genocchi numbers $G_{n}$ are obtained by setting further $x=0$. That is

$$
B_{n}(0):=B_{n} \quad E_{n}(0):=E_{n} \quad \text { and } \quad G_{n}(0):=G_{n} .
$$


In [9], Karande and Thakare obtained a class of polynomials $D_{n}(x ; u, k)$ unifying the classical Bernoulli, Euler, and Genocchi polynomials through the generating function:

$$
\frac{2^{1-k} t^{k}}{e^{t}-u} e^{x t}=\sum_{n=0}^{\infty} D_{n}(x ; u, k) \frac{t^{n}}{n !}(u \in \mathbb{R}-\{0\}, k \in \mathbb{Z}),
$$

in which

$$
D_{n}(x ; 1,1)=B_{n}(x), \quad D_{n}(x ;-1,0)=E_{n}(x), \quad \text { and } \quad 2 D_{n}(0 ;-1,1)=G_{n} .
$$

The higher order Apostol-Bernoulli polynomials $B_{n}^{(\alpha)}(x ; \lambda)$, higher order Apostol-Euler polynomials $E_{n}^{(\alpha)}(x ; \lambda)$, and higher order Apostol-Genocchi polynomials $G_{n}^{(\alpha)}(x ; \lambda)$ (see $[18,20-24,32,33])$ are defined through the generating functions:

$$
\begin{aligned}
& \left(\frac{t}{\lambda e^{t}-1}\right)^{(\alpha)} e^{x t}=\sum_{n=0}^{\infty} B_{n}^{(\alpha)}(x ; \lambda) \quad\left(|t+\ln \lambda|<2 \pi ; 1^{\alpha}=1, \alpha \in \mathbb{C}\right), \\
& \left(\frac{2}{\lambda e^{t}+1}\right)^{(\alpha)} e^{x t}=\sum_{n=0}^{\infty} E_{n}^{(\alpha)}(x ; \lambda) \quad\left(|t+\ln \lambda|<\pi ; 1^{\alpha}=1, \alpha \in \mathbb{C}\right), \\
& \left(\frac{2 t}{\lambda e^{t}+1}\right)^{(\alpha)} e^{x t}=\sum_{n=0}^{\infty} G_{n}^{(\alpha)}(x ; \lambda) \quad\left(|t+\ln \lambda|<\pi ; 1^{\alpha}=1, \alpha \in \mathbb{C}\right) .
\end{aligned}
$$

For $\lambda=1$,

$$
B_{n}^{(\alpha)}(x ; 1)=B_{n}^{\alpha}(x), \quad E_{n}^{(\alpha)}(x, 1)=E_{n}^{(\alpha)}(x), \quad \text { and } ; \quad G_{n}^{(\alpha)}(x, 1)=G_{n}^{(\alpha)}(x),
$$

where $B_{n}^{\alpha}(x), E_{n}^{\alpha}(x)$, ang $G_{n}^{\alpha}(x)$ are the Bernoulli, Euler and Genocchi polynomials of order $\alpha$, respectively. Further setting $\alpha=1$, each reduces to its classical kind.

In [26], Ozden et al. introduced a more general unification of Apostol-type Bernoulli, Euler and Genocchi polynomials via the generating function:

$$
\frac{2^{1-k} t^{k}}{\beta^{b} e^{t}-a^{b}} e^{x t}=\sum_{n=0}^{\infty} y_{n, \beta}(x ; k, a, b) \frac{t^{n}}{n !} \quad\left(|t+b \ln (\beta / a)|<2 \pi ; k \in \mathbb{N} ; a, b \in \mathbb{R}^{+} ; \beta \in \mathbb{C}\right) .
$$

This was further extended by Ozarslan [25] to higher order type of polynomials through this generating function:

$$
\left(\frac{2^{1-k} t^{k}}{\beta^{b} e^{t}-a^{b}}\right)^{\alpha} e^{x t}=\sum_{n=0}^{\infty} P_{n, \beta}^{(\alpha)}(x ; k, a, b) \frac{t^{n}}{n !} \quad\left(|t+b \ln (\beta / a)|<2 \pi ; k \in \mathbb{N} ; a, b \in \mathbb{R}^{+} ; \alpha, \beta \in \mathbb{C}\right) .
$$

Clearly,

$$
P_{n, \lambda}^{(1)}(x ; k, a, b)=y_{n, \beta}(x ; k, a, b), \quad P_{n, \lambda}^{(\alpha)}(x ; 1,1,1)=B_{n}^{(\alpha)}(x ; \lambda),
$$




$$
P_{n, \lambda}^{(\alpha)}(x ; 0,-1,1)=E_{n}^{(\alpha)}(x ; \lambda), \quad P_{n, \frac{\lambda}{2}}^{(\alpha)}\left(x ; 1,-\frac{1}{2}, 1\right)=G_{n}^{(\alpha)}(x ; \lambda) .
$$

Finally, more generalized higher order Apostol-type polynomials of parameters $a, b, c$ are defined via the generating functions (see $[1,5,7,8,28,30,31]$ ):

$$
\begin{aligned}
& \left(\frac{t}{\lambda b^{t}-a^{t}}\right)^{\alpha} c^{x t}=\sum_{n=0}^{\infty} B_{n}^{(\alpha)}(x ; a, b, c ; \lambda) \quad\left(|t \ln (b / a)+\ln \lambda|<2 \pi ; a \neq b ; 1^{\alpha}=1, \alpha \in \mathbb{C}\right), \\
& \left(\frac{2}{\lambda b^{t}+a^{t}}\right)^{\alpha} c^{x t}=\sum_{n=0}^{\infty} E_{n}^{(\alpha)}(x ; a, b, c ; \lambda) \quad\left(|t \ln (b / a)+\ln \lambda|<\pi ; 1^{\alpha}=1, \alpha \in \mathbb{C}\right), \\
& \left(\frac{2 t}{\lambda b^{t}+a^{t}}\right)^{\alpha} c^{x t}=\sum_{n=0}^{\infty} G_{n}^{(\alpha)}(x ; a, b, c ; \lambda) \quad\left(|t \ln (b / a)+\ln \lambda|<\pi ; 1^{\alpha}=1, \alpha \in \mathbb{C}\right) .
\end{aligned}
$$

In the next section, we try to unify all the previously mentioned special polynomials using a more generalized generating function.

\section{A new class of unified generalized polynomials of higher order}

Motivated by the generating relations (1), (2), and the definitions of the higher order Aposotol-type polynomials of parameters $a, b, c$, we consider the following unification of the generalized special types of polynomials mentioned in the previous section.

Definition 1. Let $a, b, c>0$, we define a unified form of generalized polynomials $\mathcal{F}_{n, k}^{(\alpha)}(x, y ; a, b, c ; \lambda)$ by means of the generating function

$$
\begin{gathered}
\left(\frac{a^{-t} t^{k}}{1-y\left(\lambda\left(\frac{b}{a}\right)^{t}-1\right)}\right)^{\alpha} c^{x t}=\sum_{n=0}^{\infty} \mathcal{F}_{n, k}^{(\alpha)}(x, y ; a, b, c ; \lambda) \frac{t^{n}}{n !}, \\
\left(\left|t \ln \left(\frac{b}{a}\right)+\ln \left(\frac{\lambda y}{y+1}\right)\right|<2 \pi ; \alpha \in \mathbb{C} ; a, b, c \in \mathbb{R}^{+} ; x, y \in \mathbb{R}, k \in \mathbb{N}_{0} ; 1^{\alpha}:=1\right) .
\end{gathered}
$$

Setting $a=1, b=e$ and $c=e$ in (3), we obtain new generalized Fubini-type polynomials $F_{n, k}^{(\alpha)}(x, y ; \lambda)$ given by the following generating function

$$
\left(\frac{t^{k}}{1-y\left(\lambda e^{t}-1\right)}\right)^{\alpha} e^{x t}=\sum_{n=0}^{\infty} F_{n, k}^{(\alpha)}(x, y ; \lambda) \frac{t^{n}}{n !} .
$$

Taking $k=0, F_{n, 0}^{(\alpha)}(x, y ; \lambda):=F_{n}^{(\alpha)}(x, y ; \lambda)$, where

$$
\left(\frac{1}{1-y\left(\lambda e^{t}-1\right)}\right)^{\alpha} e^{x t}=\sum_{n=0}^{\infty} F_{n}^{(\alpha)}(x, y ; \lambda) \frac{t^{n}}{n !} .
$$

We call $F_{n}^{(\alpha)}(x, y ; \lambda)$ as the bivariate Apostol-Fubini polynomials of order $\alpha$. Setting $\lambda=1$ in (5), we get the two-variable Fubini polynomials of higher order $F_{n}^{(\alpha)}(x, y)$ given in (1). 
Remark 1. Setting $y=-\frac{1}{2}$ and $k=0$ in (3), we obtain

$$
\mathcal{F}_{n, 0}^{(\alpha)}\left(x,-\frac{1}{2} ; a, b, c ; \lambda\right)=E_{n}^{(\alpha)}(x ; a, b, c ; \lambda) .
$$

Remark 2. Setting $y=-\frac{1}{2}$ and $k=1$ in $(3)$, we have

$$
\mathcal{F}_{n, 1}^{(\alpha)}\left(x,-\frac{1}{2} ; a, b, c ; \lambda\right)=G_{n}^{(\alpha)}(x ; a, b, c ; \lambda)
$$

Remark 3. Setting $y=-2$ and $k=1$, and replacing $\lambda$ by $\frac{\lambda}{2}$ in $(3)$, we get

$$
F_{n, 1}^{(\alpha)}\left(x,-2 ; a, b, c ; \frac{\lambda}{2}\right)=B_{n}^{(\alpha)}(x ; a, b, c ; \lambda) .
$$

Remark 4. Setting $a=1, b=c=e, \alpha=1, y=-\left(2^{k-1} u+1\right)$, and $\lambda=\frac{2^{k-1}}{2^{k-1} u+1}(u \neq 0)$; we obtain

$$
\mathcal{F}_{n, k}^{(1)}\left(x,-\left(2^{k-1} u+1\right) ; 1, e, e ; \frac{2^{k-1}}{2^{k-1} u+1}\right)=D_{n}(x ; u, k) .
$$

Remark 5. Let $a, b, \beta$ be the parameters used in (2). Setting $y=-\left(2^{k-1} a^{b}+1\right)$ and $\lambda=\frac{2^{k-1} \beta^{b}}{2^{k-1} a^{b}+1}$ in (4), we obtain, $F_{n, k}^{(\alpha)}\left(x,-\left(2^{k-1} a^{b}+1\right) ; \frac{2^{k-1} \beta^{b}}{2^{k-1} a^{b}+1}\right)=\mathcal{F}_{n, k}^{(\alpha)}\left(x,-\left(2^{k-1} a^{b}+1\right) ; 1, e, e ; \frac{2^{k-1} \beta^{b}}{2^{k-1} a^{b}+1}\right)=P_{n, \beta}^{(\alpha)}(x ; k, a, b)$.

Some of the basic properties and identities for $\mathcal{F}_{n, k}^{(\alpha)}(x, y ; a, b, c ; \lambda)$ are given in the next theorems and corollaries.

The following addition formulas are straighforward consequences of relation (3).

Theorem 1. For $\alpha, \beta, \lambda \in \mathbb{C}$ and $x, z \in \mathbb{R}$, we have

$$
\begin{aligned}
\mathcal{F}_{n, k}^{(\alpha)}(x+z, y ; a, b, c ; \lambda) & =\sum_{j=0}^{n}\left(\begin{array}{l}
n \\
j
\end{array}\right)(\ln c)^{n-j} \mathcal{F}_{j, k}^{(\alpha)}(x, y ; a, b, c ; \lambda) z^{n-j} \quad(z \neq 0) \\
& =\sum_{j=0}^{n}\left(\begin{array}{c}
n \\
j
\end{array}\right)(\ln c)^{n-j} \mathcal{F}_{j, k}^{(\alpha)}(z, y ; a, b, c ; \lambda) x^{n-j} \quad(x \neq 0), \\
\mathcal{F}_{n, k}^{(\alpha)}(x+z ; y ; a ; b ; c ; \lambda) & =\sum_{j=0}^{n}\left(\begin{array}{l}
n \\
j
\end{array}\right) \mathcal{F}_{j, k}^{(\beta)}(z, y ; a, b, c ; \lambda) \mathcal{F}_{n-j, k}^{(\alpha-\beta)}(x, y ; a, b, c ; \lambda), \\
\mathcal{F}_{n, k}^{(\alpha+\beta)}(x ; y ; a ; b ; c ; \lambda) & =\sum_{j=0}^{n}\left(\begin{array}{c}
n \\
j
\end{array}\right) \mathcal{F}_{j, k}^{(\beta)}(x, y ; a, b, c ; \lambda) \mathcal{F}_{n-j, k}^{(\alpha)}(x, y ; a, b, c ; \lambda), \\
\mathcal{F}_{n, k}^{(\alpha+\beta)}(x+z ; y ; a ; b ; c ; \lambda) & =\sum_{j=0}^{n}\left(\begin{array}{c}
n \\
j
\end{array}\right) \mathcal{F}_{j, k}^{(\alpha)}(x, y ; a, b, c ; \lambda) \mathcal{F}_{n-j, k}^{(\beta)}(z, y ; a, b, c ; \lambda)
\end{aligned}
$$




$$
=\sum_{j=0}^{n}\left(\begin{array}{c}
n \\
j
\end{array}\right) \mathcal{F}_{j, k}^{(\beta)}(x, y ; a, b, c ; \lambda) \mathcal{F}_{n-j, k}^{(\alpha)}(z, y ; a, b, c ; \lambda) .
$$

Replacing $x$ by $x-z$ in (6), (7), and (8); and replacing $\alpha$ by $\alpha-\beta$ in (9), we obtain the next results.

Corollary 1. For $\alpha, \beta, \lambda \in \mathbb{C}$ and $x, z \in \mathbb{R}$, we have

$$
\begin{aligned}
\mathcal{F}_{n, k}^{(\alpha)}(x, y ; a, b, c ; \lambda) & =\sum_{j=0}^{n}\left(\begin{array}{c}
n \\
j
\end{array}\right)(\ln c)^{n-j} \mathcal{F}_{j, k}^{(\alpha)}(x-z, y ; a, b, c ; \lambda) z^{n-j}(z \neq 0), \\
& =\sum_{j=0}^{n}\left(\begin{array}{c}
n \\
j
\end{array}\right)(\ln c)^{n-j} \mathcal{F}_{j, k}^{(\alpha)}(z, y ; a, b, c ; \lambda)(x-z)^{n-j} . \\
\mathcal{F}_{n, k}^{(\alpha)}(x, y ; a, b, c ; \lambda) & =\sum_{j=0}^{n}\left(\begin{array}{c}
n \\
j
\end{array}\right) \mathcal{F}_{j, k}^{(\alpha)}(z, y ; a, b, c ; \lambda) \mathcal{F}_{n-j, k}^{(\alpha)}(x-z, y ; a, b, c ; \lambda) \quad(z \neq x), \\
\mathcal{F}_{n, k}^{(\alpha)}(x, y ; a, b, c ; \lambda) & =\sum_{j=0}^{n}\left(\begin{array}{c}
n \\
j
\end{array}\right) \mathcal{F}_{j, k}^{(\beta)}(x, y ; a, b, c ; \lambda) \mathcal{F}_{n-j, k}^{(\alpha-\beta)}(x, y ; a, b, c ; \lambda)
\end{aligned}
$$

Setting $z=(p-1) x$ in (6), we obtain a multiplication formula for $\mathcal{F}_{n, k}^{(\alpha)}(x, y ; a, b, c ; \lambda)$.

Corollary 2. Let $p \neq 1$ and $x \neq 0$. Then

$$
\mathcal{F}_{n, k}^{(\alpha)}(p x, y ; a, b, c ; \lambda)=\sum_{j=0}^{n}\left(\begin{array}{c}
n \\
j
\end{array}\right)[(p-1) x \ln c]^{n-j} \mathcal{F}_{j, k}^{(\alpha)}(x, y ; a, b, c ; \lambda) .
$$

Theorem 2. Let $\alpha$ and $\lambda$ be arbitrary real or complex parameters. Then

$$
\begin{aligned}
\mathcal{F}_{n, k}^{(\alpha)}(-x, y ; a, b, c ; \lambda) & =(-1)^{k \alpha+n} \mathcal{F}_{n, k}^{(\alpha)}\left(x, y ; \frac{1}{a}, \frac{1}{b}, c ; \lambda\right), \\
\mathcal{F}_{n, k}^{(\alpha)}(x+\alpha, y ; a, b, c ; \lambda) & =\mathcal{F}_{n, k}^{(\alpha)}\left(x, y ; \frac{a}{c}, \frac{b}{c}, c ; \lambda\right), \\
\mathcal{F}_{n, k}^{(\alpha)}(\alpha-x, y ; a, b, c ; \lambda) & =\mathcal{F}_{n, k}^{(\alpha)}\left(-x, y ; \frac{a}{c}, \frac{b}{c}, c ; \lambda\right), \\
& =(-1)^{k \alpha+n} \mathcal{F}_{n, k}^{(\alpha)}\left(x, y ; \frac{c}{a}, \frac{c}{b}, c ; \lambda\right) .
\end{aligned}
$$
orem.

Basic differential and integral identities of $\mathcal{F}_{n, k}^{(\alpha)}(x, y ; a, b, c ; \lambda)$ are given in the next the- 
Theorem 3. Let $m, l \in \mathbb{N}_{0}$. Then for any real numbers $u$ and $v$, we have

$$
\begin{aligned}
\frac{\partial^{m}}{\partial x^{m}} \mathcal{F}_{n, k}^{(l)}(x, y ; a, b, c ; \lambda) & =\frac{n !}{(n-m) !}(\ln c)^{m} \mathcal{F}_{n-m, k}^{(l)}(x, y ; a, b, c ; \lambda), \\
\int_{u}^{v} \mathcal{F}_{n, k}^{(l)}(x, y ; a, b, c ; \lambda) d x & =\frac{1}{(n+1) \ln c}\left[\mathcal{F}_{n+1, k}^{(l)}(v, y ; a, b, c ; \lambda)-\mathcal{F}_{n+1, k}^{(l)}(u, y ; a, b, c ; \lambda)\right] .
\end{aligned}
$$

Expression (10) follows from standard arguments and induction. Moreover, (11) follows from integrating both sides of (10) with respect to $x$ (when $m=1$ ).

\section{Explicit formulas involving the Gaussian hypergeometric function}

We now establish an explicit expression of $\mathcal{F}_{n, k}^{(r)}(x, y ; a, b, c ; \lambda)$ in terms of the Gaussian hypergeometric function ${ }_{2} F_{1}(a, b ; c ; z)$ which is given by

$$
{ }_{2} F_{1}(a, b ; c ; z):=\sum_{n=0}^{\infty} \frac{(a)_{n}(b)_{n}}{(c)_{n}} \frac{z^{n}}{n !},
$$

where $c \notin \mathbb{Z}_{0} ;|z|<1 ; z=1$ and $\Re(c-a-b)>0 ; z=-1$ and $\Re(c-a-b)>-1$. Here, $(q)_{0}=1$, and $(q)_{n}=q(q+1) \cdots(q+n-1)$ for $n>0$.

Theorem 4. For $n, k, r \in \mathbb{N}_{0}$ and $y-\lambda y \neq-1$, we have

$$
\begin{aligned}
& \mathcal{F}_{n, k}^{(r)}(x, y ; a, b, c ; \lambda)=(k r) !\left(\begin{array}{c}
n \\
k r
\end{array}\right) \sum_{i=0}^{n-k r}\left(\begin{array}{c}
n-k r \\
i
\end{array}\right)\left(\begin{array}{c}
r+i-1 \\
i
\end{array}\right) \frac{\left[-\lambda y \ln \left(\frac{b}{a}\right)\right]^{i}}{(y+1-\lambda y)^{r+i}} \\
& \quad \times \sum_{m=0}^{i}(-1)^{m} m^{i}\left(\begin{array}{c}
i \\
m
\end{array}\right)\left[x \ln c-r \ln a+m \ln \left(\frac{b}{a}\right)\right]^{n-k r-i}{ }_{2} F_{1}\left(-n+k r+i, i ; 1+i ; \frac{m}{m+\frac{x \ln c-r \ln a}{\ln b-\ln a}}\right) .
\end{aligned}
$$

Proof: Note that the left hand side of (3) can be written as

$$
(y+1-\lambda y)^{-r}\left[1-\frac{\lambda y}{y+1-\lambda y}\left(e^{t \ln \left(\frac{b}{a}\right)}-1\right)\right]^{-r} t^{k r} e^{x \ln c-r \ln a} .
$$

Let $D_{t}:=\frac{d}{d t}$. Thus,

$$
\begin{aligned}
\mathcal{F}_{n, k}^{(r)}(x, y ; a, b, c ; \lambda)= & (y+1-\lambda y)^{-r} \sum_{s=0}^{n}\left(\begin{array}{l}
n \\
s
\end{array}\right)(x \ln c-r \ln a)^{n-s} \\
& \times D_{t}^{s}\left[t^{k r}\left(1-\frac{\lambda y}{y+1-\lambda y}\left[e^{t \ln \left(\frac{b}{a}\right)}-1\right]\right)^{-r}\right]_{t=0} \\
= & (y+1-\lambda y)^{-r} \sum_{s=0}^{n}\left(\begin{array}{c}
n \\
s
\end{array}\right)(x \ln c-r \ln a)^{n-s}(k r) !\left(\begin{array}{c}
s \\
k r
\end{array}\right)
\end{aligned}
$$




$$
\times D_{t}^{s-k r}\left[\left(1-\frac{\lambda y}{y+1-\lambda y}\left[e^{t \ln \left(\frac{b}{a}\right)}-1\right]\right)^{-r}\right]_{t=0} .
$$

Using

$$
(A+w)^{-r}=\sum_{i=0}^{\infty}\left(\begin{array}{c}
r+i-1 \\
i
\end{array}\right) A^{-r-i}(-w)^{i}, \quad(|w|<|A|)
$$

and

$$
\left(e^{t}-1\right)^{i}=i ! \sum_{j=1}^{\infty} S(j, i) \frac{t^{n}}{n !},
$$

where $S(j, i)$ is the Stirling numbers of the second kind, we obtain

$$
\begin{aligned}
\mathcal{F}_{n, k}^{(r)}(x, y ; a, b, c ; \lambda y)= & \sum_{s=k r}^{n}\left(\begin{array}{c}
n \\
s
\end{array}\right)(x \ln c-r \ln a)^{n-s}(k r) !\left(\begin{array}{c}
s \\
k r
\end{array}\right) \\
& \times \sum_{i=0}^{s-k r}\left(\begin{array}{c}
r+i-1 \\
i
\end{array}\right) \frac{(\lambda y)^{i}}{(y+1-\lambda y)^{r+i}}\left[\ln \left(\frac{b}{a}\right)\right]^{s-k r} i ! S(s-k r, i) .
\end{aligned}
$$

Using the explicit formula

$$
S(j, i)=\frac{1}{i !} \sum_{m=0}^{i}(-1)^{i-m}\left(\begin{array}{c}
i \\
m
\end{array}\right) m^{j}
$$

and the identity

$$
\left(\begin{array}{c}
n \\
s
\end{array}\right)\left(\begin{array}{c}
s \\
k r
\end{array}\right)=\left(\begin{array}{c}
n \\
k r
\end{array}\right)\left(\begin{array}{c}
n-k r \\
n-s
\end{array}\right)
$$

we get

$$
\begin{aligned}
\mathcal{F}_{n, k}^{(r)}(x, y ; a, b, c ; \lambda)= & \sum_{s=k r}^{n}\left(\begin{array}{c}
n \\
s
\end{array}\right)(k r) !\left(\begin{array}{c}
s \\
k r
\end{array}\right) \sum_{i=0}^{s-k r}\left(\begin{array}{c}
r+i-1 \\
i
\end{array}\right) \frac{(\lambda y)^{i}}{(y+1-\lambda y)^{r+i}} \\
& \times(x \ln c-r \ln a)^{n-s}\left[\ln \left(\frac{b}{a}\right)\right]^{s-k r} \sum_{m=0}^{i}(-1)^{i-m}\left(\begin{array}{c}
i \\
m
\end{array}\right) m^{s-k r} . \\
= & (k r) !\left(\begin{array}{c}
n \\
k r
\end{array}\right) \sum_{i=0}^{n-k r} \sum_{s=0}^{n-i-k r}\left(\begin{array}{c}
n-k r \\
n-s-k r-i
\end{array}\right)\left(\begin{array}{c}
r+i-1 \\
i
\end{array}\right)\left[\ln \left(\frac{b}{a}\right)\right]^{s+i} \\
& \frac{(-\lambda y)^{i}}{(y+1-\lambda y)^{r+i}}(x \ln c-r \ln a)^{n-s-i-k r} \sum_{m=0}^{i}(-1)^{m}\left(\begin{array}{c}
i \\
m
\end{array}\right) m^{s+i} .
\end{aligned}
$$

Using the identity

$$
(n-s-k r-i) !=\frac{(-1)^{s}(n-k r-i) !}{(-n+k r+i)_{s}},
$$


gives

$$
\begin{aligned}
\mathcal{F}_{n, k}^{(r)}(x, y ; a, b, c ; \lambda) & =(k r) !\left(\begin{array}{c}
n \\
k r
\end{array}\right) \sum_{i=0}^{n-k r}\left(\begin{array}{c}
n-k r \\
i
\end{array}\right)\left(\begin{array}{c}
r+i-1 \\
i
\end{array}\right) \frac{\left[-\lambda y \ln \left(\frac{b}{a}\right)\right]^{i}}{(y+1-\lambda y)^{r+i}} \\
& \times \sum_{m=0}^{i}(-1)^{m}\left(\begin{array}{c}
i \\
m
\end{array}\right) m^{i}(x \ln c-r \ln a)^{n-i-k r}{ }_{2} F_{1}\left(-n+k r+i, 1 ; 1+i ; \frac{-m \ln \left(\frac{b}{a}\right)}{x \ln c-r \ln a}\right) .
\end{aligned}
$$

Finally, applying Pfaff-Kummer hypergeometric transformation

${ }_{2} F_{1}(a, b ; c ; z)=(1-z)^{-a}{ }_{2} F_{1}\left(a, c-b ; c ; \frac{z}{z-1}\right) \quad\left(c \notin \mathbb{Z}_{0}^{-} ;|\arg (1-z)| \leq \pi-\epsilon(0<\epsilon<\pi)\right)$,

yields

$$
\begin{aligned}
& \mathcal{F}_{n, k}^{(r)}(x, y ; a, b, c ; \lambda)=(k r) !\left(\begin{array}{c}
n \\
k r
\end{array}\right) \sum_{i=0}^{n-k r}\left(\begin{array}{c}
n-k r \\
i
\end{array}\right)\left(\begin{array}{c}
r+i-1 \\
i
\end{array}\right) \frac{\left[-\lambda y \ln \left(\frac{b}{a}\right)\right]^{i}}{(y+1-\lambda y)^{r+i}} \\
& \quad \times \sum_{m=0}^{i}(-1)^{m}\left(\begin{array}{c}
i \\
m
\end{array}\right) m^{i}\left[x \ln c-r \ln a+m \ln \left(\frac{b}{a}\right)\right]^{n-k r-i}{ }_{2} F_{1}\left(-n+k r+i, i ; 1+i ; \frac{m}{m+\frac{x \ln c-r \ln a}{\ln b-\ln a}}\right) .
\end{aligned}
$$

Setting $y=-\frac{1}{2}$ and $k=0$ in Theorem 4 , we obtain an explict expression for $E_{n}^{(r)}(x ; a, b, c ; \lambda)$ (see Theorem 6 [31]).

Corollary 3. For $n, r \in \mathbb{N}_{0}$ and $\lambda \neq-1$, we have

$$
\begin{aligned}
& E_{n}^{(r)}(x ; a, b, c ; \lambda)=2^{r} \sum_{i=0}^{n}\left(\begin{array}{c}
n \\
i
\end{array}\right)\left(\begin{array}{c}
r+i-1 \\
i
\end{array}\right) \frac{\left[\lambda \ln \left(\frac{b}{a}\right)\right]^{i}}{(\lambda+1)^{r+i}} \\
& \quad \times \sum_{m=0}^{i}(-1)^{m} m^{i}\left(\begin{array}{c}
i \\
m
\end{array}\right)\left[x \ln c-r \ln a+m \ln \left(\frac{b}{a}\right)\right]^{n-i}{ }_{2} F_{1}\left(-n+i, i ; 1+i ; \frac{m}{m+\frac{x \ln c-r \ln a}{\ln b-\ln a}}\right) .
\end{aligned}
$$

Setting $y=-2$ and $k=1$, and replacing $\lambda$ by $\frac{\lambda}{2}$ in Theorem 4, we obtain an explicit formula for $B_{n}^{(r)}(x ; a, b, c ; \lambda)$ (see Theorem $6[30]$ ).

Corollary 4. For $n, r \in \mathbb{N}_{0}$ and $\lambda \neq 1$, we have

$$
\begin{aligned}
& B_{n}^{(r)}(x ; a, b, c ; \lambda)=r !\left(\begin{array}{c}
n \\
r
\end{array}\right) \sum_{i=0}^{n-r}\left(\begin{array}{c}
n-r \\
i
\end{array}\right)\left(\begin{array}{c}
r+i-1 \\
i
\end{array}\right) \frac{\left[\lambda \ln \left(\frac{b}{a}\right)\right]^{i}}{(\lambda-1)^{r+i}} \\
& \quad \times \sum_{m=0}^{i}(-1)^{m} m^{i}\left(\begin{array}{c}
i \\
m
\end{array}\right)\left[x \ln c-r \ln a+m \ln \left(\frac{b}{a}\right)\right]^{n-r-i}{ }_{2} F_{1}\left(-n+i, i ; 1+i ; \frac{m}{m+\frac{x \ln c-r \ln a}{\ln b-\ln a}}\right) .
\end{aligned}
$$


Setting $y=-\frac{1}{2}$ and $k=1$ in Theorem 4 , we obtain an explicit formula for $G_{n}^{(r)}(x ; a, b, c ; \lambda)$ (see Theorem 9 [31]).

Corollary 5. For $n, r \in \mathbb{N}_{0}$, and $\lambda \neq-1$, we have

$$
\begin{aligned}
& G_{n}^{(r)}(x ; a, b, c ; \lambda)=2^{r} r !\left(\begin{array}{c}
n \\
r
\end{array}\right) \sum_{i=0}^{n-r}\left(\begin{array}{c}
n-r \\
i
\end{array}\right)\left(\begin{array}{c}
r+i-1 \\
i
\end{array}\right) \frac{\left[\lambda \ln \left(\frac{b}{a}\right)\right]^{i}}{(\lambda+1)^{r+i}} \\
& \quad \times \sum_{m=0}^{i}(-1)^{m} m^{i}\left(\begin{array}{c}
i \\
m
\end{array}\right)\left[x \ln c-r \ln a+m \ln \left(\frac{b}{a}\right)\right]^{n-r-i}{ }_{2} F_{1}\left(-n+i, i ; 1+i ; \frac{m}{m+\frac{x \ln c-r \ln a}{\ln b-\ln a}}\right) .
\end{aligned}
$$

Taking $a=1$ and $b=c=e$ Theorem 4 , we get an explicit formula of $F_{n, k}^{(r)}(x, y ; \lambda)$.

Corollary 6. For $n, k, r \in \mathbb{N}_{0}$ and $y-\lambda y \neq-1$, we have

$$
\begin{aligned}
F_{n, k}^{(r)}(x, y ; \lambda) & =(k r) !\left(\begin{array}{c}
n \\
k r
\end{array}\right) \sum_{i=0}^{n-k r}\left(\begin{array}{c}
n-k r \\
i
\end{array}\right)\left(\begin{array}{c}
r+i-1 \\
i
\end{array}\right) \frac{(-\lambda y)^{i}}{(y+1-\lambda y)^{r+i}} \\
& \times \sum_{m=0}^{i}(-1)^{m}\left(\begin{array}{c}
i \\
m
\end{array}\right) m^{i}(x+m)^{n-k r-i}{ }_{2} F_{1}\left(-n+k r+i, i ; 1+i ; \frac{m}{m+x}\right) .
\end{aligned}
$$

Setting $y=-\left(2^{k-1} a^{b}+1\right)$ and $\lambda=\frac{2^{k-1} \beta^{b}}{2^{k-1} a^{b}+1}$ in Corollary 6 , we obtain an explicit formula of $P_{n, \beta}^{(r)}(x ; a, b)$ (see Theorem $2.1[25]$ ).

Corollary 7. For $n, k, r \in \mathbb{N}_{0}, \quad a, b \in \mathbb{R}^{+}, \beta \neq a$,

$$
\begin{aligned}
P_{n, \beta}^{(r)}(x ; a, b)= & 2^{(1-k) r}(k r) !\left(\begin{array}{c}
n \\
k r
\end{array}\right) \sum_{i=0}^{n-k r}\left(\begin{array}{c}
n-k r \\
i
\end{array}\right)\left(\begin{array}{c}
r+i-1 \\
i
\end{array}\right) \frac{\beta^{b i}}{\left(\beta^{a}-a^{b}\right)^{r+i}} \\
& \times \sum_{m=0}^{i}(-1)^{m}\left(\begin{array}{c}
i \\
m
\end{array}\right) m^{i}(x+m)^{n-k r-i}{ }_{2} F_{1}\left(-n+k r+i, i ; 1+i ; \frac{m}{m+x}\right) .
\end{aligned}
$$

\section{Symmetry Identities}

In this section, we derive and investigate some symmetry identities for $\mathcal{F}_{n, k}^{(r)}(x, y ; a, b, c ; \lambda)$.

For each $k \in \mathbb{N}_{0}$, the sum of integer powers $S_{k}(n)$ is defined by $S_{k}(n)=\sum_{j=0}^{n-1} j^{k}$ and has the exponential generating function

$$
\sum_{k=0}^{\infty} S_{k}(n) \frac{t^{k}}{k !}=\frac{e^{n t}-1}{e^{t}-1}
$$


In [19], Lu and Srivastava defined the generalized sum of integer powers $S_{k}(n ; \lambda)$ through the generating function

$$
\sum_{k=0}^{\infty} S_{k}(n ; \lambda) \frac{t^{k}}{k !}=\frac{\lambda e^{n t}-1}{\lambda e^{t}-1} \quad(\lambda \in \mathbb{C}) .
$$

Clearly, $S_{k}(n ; 1)=S_{k}(n)$.

Definition 2. Let $\lambda$ be any real or complex paramete and $b>0$, we define a more generalized sum of integer powers $S_{k}(n ; b, \lambda)$ using the generating function

$$
\sum_{k=0}^{\infty} S_{k}(n ; b, \lambda) \frac{t^{k}}{k !}=\frac{\lambda b^{n t}-1}{\lambda b^{t}-1}
$$

Obviously, $S_{k}(n ; e, \lambda)=S_{k}(n ; \lambda)$ and $S_{k}(n ; e, 1)=S_{k}(n)$.

Now, we establish some symmetry identities involving these new class of unified generalized polynomials. The techniques used in here are parallel to the methods in $[25,33]$. Thus, we also include some results in [25] as corollaries.

Theorem 5. For $u, v, m \in \mathbb{N} ; n \in \mathbb{N}_{0}$; and $y \neq-1$, we have

$$
\begin{aligned}
& \sum_{r=0}^{n}\left(\begin{array}{l}
n \\
r
\end{array}\right) u^{n-r} v^{r+k} \mathcal{F}_{n-r, k}^{(m)}\left(v x-\frac{v}{u} \log _{c} a, y ; a, b, c ; \lambda\right) \sum_{l=0}^{r}\left(\begin{array}{l}
r \\
l
\end{array}\right) S_{l}\left(u-1, \frac{b}{a} ; \frac{\lambda y}{y+1}\right) \mathcal{F}_{r-l, k}^{(m-1)}(u z, y ; a, b, c ; \lambda) \\
& =\sum_{r=0}^{n}\left(\begin{array}{l}
n \\
r
\end{array}\right) v^{n-r} u^{r+k} \mathcal{F}_{n-r, k}^{(m)}\left(u x-\frac{u}{v} \log _{c} a, y ; a, b, c ; \lambda\right) \sum_{l=0}^{r}\left(\begin{array}{l}
r \\
l
\end{array}\right) S_{l}\left(v-1, \frac{b}{a} ; \frac{\lambda y}{y+1}\right) \mathcal{F}_{r-l, k}^{(m-1)}(v z, y ; a, b, c ; \lambda) .
\end{aligned}
$$

Proof: Let

$$
G(t):=\frac{t^{2 k m-k} a^{-m(u+v) t} c^{u v x t}\left(1-y\left(\lambda\left(\frac{b}{a}\right)^{u v t}-1\right)\right) c^{u v z t}}{\left(1-y\left(\lambda\left(\frac{b}{a}\right)^{u t}-1\right)\right)^{m}\left(1-y\left(\lambda\left(\frac{b}{a}\right)^{v t}-1\right)\right)^{m}} .
$$

Grouping factors and expanding $G(t)$ into series, we obtain

$$
\begin{aligned}
G(t)= & \frac{1}{u^{k m} v^{k(m-1)}}\left(\frac{a^{-u t}(u t)^{k}}{1-y\left(\lambda\left(\frac{b}{a}\right)^{u t}-1\right)}\right)^{m} c^{v x(u t)} a^{-v t} \\
& \times\left(\frac{\frac{\lambda y}{y+1}\left(\frac{b}{a}\right)^{u v t}-1}{\frac{\lambda y}{y+1}\left(\frac{b}{a}\right)^{v t}-1}\right)\left(\frac{a^{-v t}(v t)^{k}}{1-y\left(\lambda\left(\frac{b}{a}\right)^{v t}-1\right)}\right)^{m-1} c^{u z(v t)} \\
= & \frac{1}{u^{k m} v^{k(m-1)}} \sum_{n=0}^{\infty} \mathcal{F}_{n, k}^{(m)}\left(v x-\frac{v}{u} \log _{c} a, y ; a, b, c ; \lambda\right) \frac{(u t)^{n}}{n !}
\end{aligned}
$$




$$
\begin{gathered}
\times \sum_{n=0}^{\infty} S_{n}\left(u-1, \frac{b}{a} ; \frac{\lambda y}{y+1}\right) \frac{(v t)^{n}}{n !} \cdot \sum_{n=0}^{\infty} \mathcal{F}_{n, k}^{(m-1)}(u z, y ; a, b, c ; \lambda) \frac{(v t)^{n}}{n !} \\
=\frac{1}{(u v)^{k m}} \sum_{n=0}^{\infty}\left[\sum_{r=0}^{n}\left(\begin{array}{l}
n \\
r
\end{array}\right) u^{n-r} v^{r+k} \mathcal{F}_{n-r, k}^{(m)}\left(v x-\frac{v}{u} \log _{c} a, y ; a, b, c ; \lambda\right)\right. \\
\left.\times \sum_{l=0}^{r}\left(\begin{array}{l}
r \\
l
\end{array}\right) S_{l}\left(u-1, \frac{b}{a} ; \frac{\lambda y}{y+1}\right) \mathcal{F}_{r-l, k}^{(m-1)}(u z, y ; a, b, c ; \lambda)\right] \frac{t^{n}}{n !} .
\end{gathered}
$$

Similarly,

$$
\begin{aligned}
G(t)= & \frac{1}{v^{k m} u^{k(m-1)}}\left(\frac{(v t)^{k}}{1-y\left(\lambda\left(\frac{b}{a}\right)^{v t}-1\right)}\right)^{m} c^{u x(v t)} a^{-u t} \\
& \times\left(\frac{\frac{\lambda y}{y+1}\left(\frac{b}{a}\right)^{u v t}-1}{\frac{\lambda y}{y+1}\left(\frac{b}{a}\right)^{u t}-1}\right)\left(\frac{(u t)^{k}}{1-y\left(\lambda\left(\frac{b}{a}\right)^{u t}-1\right)}\right)^{m-1} c^{v z(u t)} \\
= & \frac{1}{v^{k m} u^{k(m-1)}} \sum_{n=0}^{\infty} \mathcal{F}_{n, k}^{(m)}\left(u x-\frac{u}{v} \log _{c} a, y ; a, b, c ; \lambda\right) \frac{(v t)^{n}}{n !} \\
& \times \sum_{n=0}^{\infty} S_{n}\left(v-1, \frac{b}{a} ; \frac{\lambda y}{y+1}\right) \frac{(u t)^{n}}{n !} \cdot \sum_{n=0}^{\infty} \mathcal{F}_{n, k}^{(m-1)}(v z, y ; a, b, c ; \lambda) \frac{(u t)^{n}}{n !} \\
= & \frac{1}{(v u)^{k m}} \sum_{n=0}^{\infty}\left[\sum_{r=0}^{n}\left(\begin{array}{l}
n \\
r
\end{array}\right) v^{n-r} u^{r+k} \mathcal{F}_{n-r, k}^{(m)}\left(u x-\frac{u}{v} \log _{c} a, y ; a, b, c ; \lambda\right)\right. \\
& \left.\times \sum_{l=0}^{r}\left(\begin{array}{l}
r \\
l
\end{array}\right) S_{l}\left(v-1, \frac{b}{a} ; \frac{\lambda y}{y+1}\right) \mathcal{F}_{r-l, k}^{(m-1)}(v z, y ; a, b, c ; \lambda)\right] \frac{t^{n}}{n !} .
\end{aligned}
$$

Comparing the (12) and (13) yields the desired result.

Setting $y=-\frac{1}{2}$ and $k=0$ in Theorem 5 , we have a symmetry identity for $E_{n}^{(\alpha)}(x ; a, b, c ; \lambda)$.

Corollary 8. For $u, v, m \in \mathbb{N}$ and $n \in \mathbb{N}_{0}$, we have

$$
\begin{aligned}
& \sum_{r=0}^{n}\left(\begin{array}{l}
n \\
r
\end{array}\right) u^{n-r} v^{r} E_{n-r}^{(m)}\left(v x-\frac{v}{u} \log _{c} a ; a, b, c ; \lambda\right) \sum_{l=0}^{r}\left(\begin{array}{l}
r \\
l
\end{array}\right) S_{l}\left(u-1, \frac{b}{a} ;-\lambda\right) E_{r-l}^{(m-1)}(u z ; a, b, c ; \lambda) \\
& =\sum_{r=0}^{n}\left(\begin{array}{l}
n \\
r
\end{array}\right) v^{n-r} u^{r} E_{n-r}^{(m)}\left(u x-\frac{u}{v} \log _{c} a ; a, b, c ; \lambda\right) \sum_{l=0}^{r}\left(\begin{array}{l}
r \\
l
\end{array}\right) S_{l}\left(v-1, \frac{b}{a} ;-\lambda\right) E_{r-l}^{(m-1)}(v z ; a, b, c ; \lambda) .
\end{aligned}
$$

Setting $y=-2$ and $k=1$, and replacing $\lambda$ by $\frac{\lambda}{2}$ in Theorem 5 , we have a symmetry identity for $B_{n}^{(\alpha)}(x ; a, b, c ; \lambda)$. 
Corollary 9. For $u, v, m \in \mathbb{N}$ and $n \in \mathbb{N}_{0}$, we have

$$
\begin{aligned}
& \sum_{r=0}^{n}\left(\begin{array}{l}
n \\
r
\end{array}\right) u^{n-r} v^{r+1} B_{n-r}^{(m)}\left(v x-\frac{v}{u} \log _{c} a ; a, b, c ; \lambda\right) \sum_{l=0}^{r}\left(\begin{array}{l}
r \\
l
\end{array}\right) S_{l}\left(u-1, \frac{b}{a} ; \lambda\right) B_{r-l}^{(m-1)}(u z ; a, b, c ; \lambda) \\
& =\sum_{r=0}^{n}\left(\begin{array}{l}
n \\
r
\end{array}\right) v^{n-r} u^{r+1} B_{n-r}^{(m)}\left(u x-\frac{u}{v} \log _{c} a ; a, b, c ; \lambda\right) \sum_{l=0}^{r}\left(\begin{array}{l}
r \\
l
\end{array}\right) S_{l}\left(v-1, \frac{b}{a} ; \lambda\right) B_{r-l}^{(m-1)}(v z ; a, b, c ; \lambda) .
\end{aligned}
$$

Setting $y=-\frac{1}{2}$ and $k=0$ in Theorem 5 , we have a symmetry identity for $G_{n}^{(\alpha)}(x ; a, b, c ; \lambda)$.

Corollary 10. For $u, v, m \in \mathbb{N}$ and $n \in \mathbb{N}_{0}$, we have

$$
\begin{aligned}
& \sum_{r=0}^{n}\left(\begin{array}{l}
n \\
r
\end{array}\right) u^{n-r} v^{r+1} G_{n-r}^{(m)}\left(v x-\frac{v}{u} \log _{c} a ; a, b, c ; \lambda\right) \sum_{l=0}^{r}\left(\begin{array}{l}
r \\
l
\end{array}\right) S_{l}\left(u-1, \frac{b}{a} ;-\lambda\right) G_{r-l}^{(m-1)}(u z ; a, b, c ; \lambda) \\
& =\sum_{r=0}^{n}\left(\begin{array}{l}
n \\
r
\end{array}\right) v^{n-r} u^{r+1} G_{n-r}^{(m)}\left(u x-\frac{u}{v} \log _{c} a ; a, b, c ; \lambda\right) \sum_{l=0}^{r}\left(\begin{array}{l}
r \\
l
\end{array}\right) S_{l}\left(v-1, \frac{b}{a} ;-\lambda\right) G_{r-l}^{(m-1)}(v z ; a, b, c ; \lambda) .
\end{aligned}
$$

Setting $b=c=e$ and $a=1$ in Theorem 5, we obtain a symmetry identity for the higher order generalized Fubini-type polynomials $F_{n, k}^{(\alpha)}(x, y ; \lambda)$.

Corollary 11. For $u, v, m \in \mathbb{N}$ and $n \in \mathbb{N}_{0}$, we have

$$
\begin{aligned}
\sum_{r=0}^{n}\left(\begin{array}{l}
n \\
r
\end{array}\right) u^{n-r} v^{r+k} F_{n-r, k}^{(m)}(v x, y ; \lambda) \sum_{l=0}^{r}\left(\begin{array}{l}
r \\
l
\end{array}\right) S_{l}\left(u-1 ; \frac{\lambda y}{y+1}\right) F_{r-l, k}^{(m-1)}(u z, y ; \lambda) \\
\quad=\sum_{r=0}^{n}\left(\begin{array}{l}
n \\
r
\end{array}\right) v^{n-r} u^{r+k} F_{n-r, k}^{(m)}(u x, y ; \lambda) \sum_{l=0}^{r}\left(\begin{array}{l}
r \\
l
\end{array}\right) S_{l}\left(v-1 ; \frac{\lambda y}{y+1}\right) F_{r-l, k}^{(m-1)}(v z, y ; \lambda) .
\end{aligned}
$$

Setting $y=-\left(2^{k-1} a^{b}+1\right)$ and $\lambda=\frac{2^{k-1} \beta^{b}}{2^{k-1}+1}$ in Corollary 11, we get Theorem 3.1 of [25].

Corollary 12. For $a, b \in \mathbb{R}-\{0\} ; \beta \in C ; u, v, m \in \mathbb{N}$ and $n \in \mathbb{N}_{0}$, we have

$$
\begin{aligned}
& \sum_{r=0}^{n}\left(\begin{array}{l}
n \\
r
\end{array}\right) u^{n-r} v^{r+k} P_{n-r, \beta}^{(m)}(v x ; k, a, b) \sum_{l=0}^{r}\left(\begin{array}{l}
r \\
l
\end{array}\right) S_{l}\left(u-1 ;\left(\frac{\beta}{a}\right)^{b}\right) P_{r-l, \beta}^{(m-1)}(u z ; k, a, b) \\
& =\sum_{r=0}^{n}\left(\begin{array}{c}
n \\
r
\end{array}\right) v^{n-r} u^{r+k} P_{n-r, \beta}^{(m)}(u x ; k, a, b) \sum_{l=0}^{r}\left(\begin{array}{l}
r \\
l
\end{array}\right) S_{l}\left(v-1 ;\left(\frac{\beta}{a}\right)^{b}\right) P_{r-l, \beta}^{(m-1)}(v z ; k, a, b) .
\end{aligned}
$$

Theorem 6. For $u, v, m \in \mathbb{N}$ and $n \in \mathbb{N}_{0}$, we have

$$
\begin{aligned}
& \sum_{r=0}^{n}\left(\begin{array}{c}
n \\
r
\end{array}\right) \sum_{i=0}^{u-1} \sum_{j=0}^{v-1}\left(\frac{\lambda y}{y+1}\right)^{i+j} u^{r} v^{n-r} \mathcal{F}_{r, k}^{(m)}\left(v x+\frac{v}{u} i \log _{c}(b / a), y ; a, b, c ; \lambda\right) \mathcal{F}_{n-r, k}^{(m)}\left(u z+\frac{u}{v} j \log _{c}(b / a), y ; a, b, c ; \lambda\right) \\
& =\sum_{r=0}^{n}\left(\begin{array}{l}
n \\
r
\end{array}\right) \sum_{i=0}^{v-1} \sum_{j=0}^{u-1}\left(\frac{\lambda y}{y+1}\right)^{i+j} v^{r} u^{n-r} \mathcal{F}_{r, k}^{(m)}\left(u x+\frac{u}{v} i \log _{c}(b / a), y ; a, b, c ; \lambda\right) \mathcal{F}_{n-r, k}^{(m)}\left(v z+\frac{v}{u} j \log _{c}(b / a), y ; a, b, c ; \lambda\right) .
\end{aligned}
$$


Proof: Consider

$$
H(t)=\frac{t^{2 k m} a^{-t(u+v) m} c^{u v x t}\left(-(\lambda y)^{u}\left(\frac{b}{a}\right)^{u v t}+(y+1)^{u}\right)\left(-(\lambda y)^{v}\left(\frac{b}{a}\right)^{u v t}+(y+1)^{v}\right) c^{u v z t}}{\left(1-y\left(\lambda\left(\frac{b}{a}\right)^{u t}-1\right)\right)^{m+1}\left(1-y\left(\lambda\left(\frac{b}{a}\right)^{v t}-1\right)\right)^{m+1}} .
$$

Expanding $H(t)$ into series, we have

$$
\begin{aligned}
H(t)= & \frac{(y+1)^{u-1}(y+1)^{v-1}}{(u v)^{k m}}\left(\frac{a^{-u t}(u t)^{k}}{1-y\left(\lambda\left(\frac{b}{a}\right)^{u t}-1\right)}\right)^{m} c^{v x(u t)}\left(\frac{\left(\frac{\lambda y}{y+1}\right)^{u}\left(\frac{b}{a}\right)^{u v t}-1}{\frac{\lambda y}{y+1}\left(\frac{b}{a}\right)^{v t}-1}\right) \\
& \times\left(\frac{a^{-v t}(v t)^{k}}{1-y\left(\lambda\left(\frac{b}{a}\right)^{v t}-1\right)}\right)^{m} c^{u z(v t)}\left(\frac{\left(\frac{\lambda y}{y+1}\right)^{v}\left(\frac{b}{a}\right)^{u v t}-1}{\frac{\lambda y}{y+1}\left(\frac{b}{a}\right)^{u t}-1}\right) \cdot \\
= & \frac{(y+1)^{u-1}(y+1)^{v-1}}{(u v)^{k m}} \sum_{i=0}^{u-1}\left(\frac{\lambda y}{y+1}\right)^{i}\left(\frac{b}{a}\right)^{i v t}\left(\frac{a^{-u t}(u t)^{k}}{1-y\left(\lambda\left(\frac{b}{a}\right)^{u t}-1\right)}\right)^{m} c^{v x(u t)} \\
& \times \sum_{j=0}^{v-1}\left(\frac{\lambda y}{y+1}\right)^{j}\left(\frac{b}{a}\right)^{j u t}\left(\frac{a^{-v t}(v t)^{k}}{1-y\left(\lambda\left(\frac{b}{a}\right)^{v t}-1\right)}\right)^{m} c^{u z(v t)} \\
= & \frac{(y+1)^{u+v-2}}{(u v)^{k m}} \sum_{n=0}^{\infty}\left[\sum_{r=0}^{n}\left(\begin{array}{l}
n \\
r
\end{array}\right) \sum_{i=0}^{u-1} \sum_{j=0}^{v-1}\left(\frac{\lambda y}{y+1}\right)^{i+j} u^{r} v^{n-r} \mathcal{F}_{r, k}^{(m)}\left(v x+\frac{v}{u} i \log _{c}(b / a), y ; a, b, c ; \lambda\right)\right. \\
& \left.\times \mathcal{F}_{n-r, k}^{(m)}\left(u z+\frac{u}{v} j \log _{c}(b / a), y ; a, b, c ; \lambda\right)\right] \frac{t^{n}}{n !} .
\end{aligned}
$$

Similarly,

$$
\begin{aligned}
H(t)= & \frac{(y+1)^{u-1}(y+1)^{v-1}}{(u v)^{k m}}\left(\frac{a^{-v t}(v t)^{k}}{1-y\left(\lambda\left(\frac{b}{a}\right)^{v t}-1\right)}\right)^{m} c^{u x(v t)}\left(\frac{\left(\frac{\lambda y}{y+1}\right)^{v}\left(\frac{b}{a}\right)^{u v t}-1}{\frac{\lambda y}{y+1}\left(\frac{b}{a}\right)^{u t}-1}\right) \\
& \times\left(\frac{a^{-u t}(u t)^{k}}{1-y\left(\lambda\left(\frac{b}{a}\right)^{u t}-1\right)}\right)^{m} c^{v z(u t)}\left(\frac{\left(\frac{\lambda y}{y+1}\right)^{u}\left(\frac{b}{a}\right)^{u v t}-1}{\frac{\lambda y}{y+1}\left(\frac{b}{a}\right)^{v t}-1}\right) \cdot \\
= & \frac{(y+1)^{u-1}(y+1)^{v-1}}{(u v)^{k m}} \sum_{i=0}^{v-1}\left(\frac{\lambda y}{y+1}\right)^{i}\left(\frac{b}{a}\right)^{i u t}\left(\frac{a^{-v t}(v t)^{k}}{1-y\left(\lambda\left(\frac{b}{a}\right)^{v t}-1\right)}\right)^{m} c^{u x(v t)} \\
& \times \sum_{j=0}^{u-1}\left(\frac{\lambda y}{y+1}\right)^{j}\left(\frac{b}{a}\right)^{j v t}\left(\frac{a^{-u t}(u t)^{k}}{1-y\left(\lambda\left(\frac{b}{a}\right)^{u t}-1\right)}\right)^{m} c^{v z(u t)} \\
= & \frac{(y+1)^{u+v-2}}{(u v)^{k m}} \sum_{n=0}^{\infty}\left[\sum_{r=0}^{n}\left(\begin{array}{c}
n \\
r
\end{array}\right) \sum_{i=0}^{v-1} \sum_{j=0}^{u-1}\left(\frac{\lambda y}{y+1}\right)^{i+j} v^{r} u^{n-r} \mathcal{F}_{r, k}^{(m)}\left(u x+\frac{u}{v} i \log _{c}(b / a), y ; a, b, c ; \lambda\right)\right.
\end{aligned}
$$


N. G. Acala / Eur. J. Pure Appl. Math, 13 (3) (2020), 587-607

$$
\left.\times \mathcal{F}_{n-r, k}^{(m)}\left(v z+\frac{v}{u} j \log _{c}(b / a), y ; a, b, c ; \lambda\right)\right] \frac{t^{n}}{n !} .
$$

Comparing (14) and (15), we get the desired result.

Setting $y=-\frac{1}{2}$ and $k=0$ in Theorem 6 , we obtain the following corollary.

Corollary 13. For $u, v, m \in \mathbb{N}$ and $n \in \mathbb{N}_{0}$, we have

$$
\begin{aligned}
& \sum_{r=0}^{n}\left(\begin{array}{l}
n \\
r
\end{array}\right) \sum_{i=0}^{u-1} \sum_{j=0}^{v-1}(-\lambda)^{i+j} u^{r} v^{n-r} E_{r}^{(m)}\left(v x+\frac{v}{u} i \log _{c}(b / a) ; a, b, c ; \lambda\right) E_{n-r}^{(m)}\left(u z+\frac{u}{v} j \log _{c}(b / a) ; a, b, c ; \lambda\right) \\
& =\sum_{r=0}^{n}\left(\begin{array}{l}
n \\
r
\end{array}\right) \sum_{i=0}^{v-1} \sum_{j=0}^{u-1}(-\lambda)^{i+j} v^{r} u^{n-r} E_{r}^{(m)}\left(u x+\frac{u}{v} i \log _{c}(b / a) ; a, b, c ; \lambda\right) E_{n-r}^{(m)}\left(v z+\frac{v}{u} j \log _{c}(b / a) ; a, b, c ; \lambda\right) .
\end{aligned}
$$

Setting $y=-2, k=0$ and replacing $\lambda$ by $\frac{\lambda}{2}$ in Theorem 6 , we obtain the following corollary.

Corollary 14. For $u, v, m \in \mathbb{N}$ and $n \in \mathbb{N}_{0}$, we have

$$
\begin{aligned}
& \sum_{r=0}^{n}\left(\begin{array}{l}
n \\
r
\end{array}\right) \sum_{i=0}^{u-1} \sum_{j=0}^{v-1}(\lambda)^{i+j} u^{r} v^{n-r} B_{r}^{(m)}\left(v x+\frac{v}{u} i \log _{c}(b / a) ; a, b, c ; \lambda\right) B_{n-r}^{(m)}\left(u z+\frac{u}{v} j \log _{c}(b / a) ; a, b, c ; \lambda\right) \\
& =\sum_{r=0}^{n}\left(\begin{array}{c}
n \\
r
\end{array}\right) \sum_{i=0}^{v-1} \sum_{j=0}^{u-1}(\lambda)^{i+j} v^{r} u^{n-r} B_{r}^{(m)}\left(u x+\frac{u}{v} i \log _{c}(b / a) ; a, b, c ; \lambda\right) B_{n-r}^{(m)}\left(v z+\frac{v}{u} j \log _{c}(b / a) ; a, b, c ; \lambda\right) .
\end{aligned}
$$

Setting $y=-\frac{1}{2}$ and $k=1$ in Theorem 6 , we obtain the following corollary.

Corollary 15. For $u, v, m \in \mathbb{N}$ and $n \in \mathbb{N}_{0}$, we have

$$
\begin{aligned}
& \sum_{r=0}^{n}\left(\begin{array}{l}
n \\
r
\end{array}\right) \sum_{i=0}^{u-1} \sum_{j=0}^{v-1}(-\lambda)^{i+j} u^{r} v^{n-r} G_{r}^{(m)}\left(v x+\frac{v}{u} i \log _{c}(b / a) ; a, b, c ; \lambda\right) G_{n-r}^{(m)}\left(u z+\frac{u}{v} j \log _{c}(b / a) ; a, b, c ; \lambda\right) \\
& =\sum_{r=0}^{n}\left(\begin{array}{l}
n \\
r
\end{array}\right) \sum_{i=0}^{v-1} \sum_{j=0}^{u-1}(-\lambda)^{i+j} v^{r} u^{n-r} G_{r}^{(m)}\left(u x+\frac{u}{v} i \log _{c}(b / a) ; a, b, c ; \lambda\right) G_{n-r}^{(m)}\left(v z+\frac{v}{u} j \log _{c}(b / a) ; a, b, c ; \lambda\right) .
\end{aligned}
$$

Setting $a=1$ and $b=c=e$ in Theorem 6 , we obtain another symmetry identity for the higher order bivariate Fubini-type polynomials $F_{n, k}^{(\alpha)}(x, y ; \lambda)$. 
Corollary 16. For $u, v, m \in \mathbb{N}$ and $n \in \mathbb{N}_{0}$, we have

$$
\begin{aligned}
& \sum_{r=0}^{n}\left(\begin{array}{l}
n \\
r
\end{array}\right) \sum_{i=0}^{u-1} \sum_{j=0}^{v-1}\left(\frac{\lambda y}{y+1}\right)^{i+j} u^{r} v^{n-r} F_{r, k}^{(m)}\left(v x+\frac{v}{u} i, y ; \lambda\right) F_{n-r, k}^{(m)}\left(u z+\frac{u}{v} j, y ; \lambda\right) \\
& \quad=\sum_{r=0}^{n}\left(\begin{array}{l}
n \\
r
\end{array}\right) \sum_{i=0}^{v-1} \sum_{j=0}^{u-1}\left(\frac{\lambda y}{y+1}\right)^{i+j} v^{r} u^{n-r} F_{r, k}^{(m)}\left(u x+\frac{u}{v} i, y ; \lambda\right) F_{n-r, k}^{(m)}\left(v z+\frac{v}{u} j, y ; \lambda\right) .
\end{aligned}
$$

Taking $y=-\left(2^{k-1} a^{b}+1\right)$ and $\lambda=\frac{2^{k-1} \beta^{b}}{2^{k-1}+1}$ in Corollary 16 , we get Theorem 3.5 of [25].

Corollary 17. For $a, b>0 ; \beta \in C ; u, v, m \in \mathbb{N}$ and $n \in \mathbb{N}_{0}$, we have

$$
\begin{aligned}
& \sum_{r=0}^{n}\left(\begin{array}{l}
n \\
r
\end{array}\right) \sum_{i=0}^{u-1} \sum_{j=0}^{v-1}\left(\frac{\beta}{a}\right)^{b(i+j)} u^{r} v^{n-r} P_{r, \beta}^{(m)}\left(v x+\frac{v}{u} i ; k, a, b\right) P_{n-r, \beta}^{(m)}\left(u z+\frac{u}{v} j ; k, a, b\right) \\
& \quad=\sum_{r=0}^{n}\left(\begin{array}{l}
n \\
r
\end{array}\right) \sum_{i=0}^{v-1} \sum_{j=0}^{u-1}\left(\frac{\beta}{a}\right)^{b(i+j)} v^{r} u^{n-r} P_{r, \beta}^{(m)}\left(u x+\frac{u}{v} i ; k, a, b\right) p_{n-r, k}^{(m)}\left(v z+\frac{v}{u} j ; k, a, b\right) .
\end{aligned}
$$

Theorem 7. For $u, v, m \in \mathbb{N}, n \in \mathbb{N}_{0}$ and $y \neq-1$, we have

$$
\begin{aligned}
& \sum_{r=0}^{n}\left(\begin{array}{l}
n \\
r
\end{array}\right) \sum_{i=0}^{u-1} \sum_{j=0}^{v-1}\left(\frac{\lambda y}{y+1}\right)^{i+j} u^{r} v^{n-r} \mathcal{F}_{r, k}^{(m)}\left(v x+\left(i \frac{v}{u}+j\right) \log _{c}(b / a), y ; a, b, c ; \lambda\right) \mathcal{F}_{n-r, k}^{(m)}(u z, y ; a, b, c ; \lambda) \\
& =\sum_{r=0}^{n}\left(\begin{array}{l}
n \\
r
\end{array}\right) \sum_{i=0}^{v-1} \sum_{j=0}^{u-1}\left(\frac{\lambda y}{y+1}\right)^{i+j} v^{r} u^{n-r} \mathcal{F}_{r, k}^{(m)}\left(u x+\left(i \frac{u}{v}+j\right) \log _{c}(b / a), y ; a, b, c ; \lambda\right) \mathcal{F}_{n-r, k}^{(m)}(v z, y ; a, b, c ; \lambda) .
\end{aligned}
$$

Proof: Consider

$$
L(t)=\frac{t^{2 k m} a^{-t(u+v) m} c^{u v x t}\left(-(\lambda y)^{u}\left(\frac{b}{a}\right)^{u v t}+(y+1)^{u}\right)\left(-(\lambda y)^{v}\left(\frac{b}{a}\right)^{u v t}+(y+1)^{v}\right) c^{u v z t}}{\left(1-y\left(\lambda\left(\frac{b}{a}\right)^{u t}-1\right)\right)^{m+1}\left(1-y\left(\lambda\left(\frac{b}{a}\right)^{v t}-1\right)\right)^{m+1}} .
$$

Expanding $L(t)$ into a series, we get

$$
\begin{aligned}
L(t)= & \frac{(y+1)^{u-1}(y+1)^{v-1}}{(u v)^{k m}}\left(\frac{a^{-u t}(u t)^{k}}{1-y\left(\lambda\left(\frac{b}{a}\right)^{u t}-1\right)}\right)^{m} c^{u v x t t}\left(\frac{\left(\frac{\lambda y}{y+1}\right)^{u}\left(\frac{b}{a}\right)^{u v t}-1}{\frac{\lambda y}{y+1}\left(\frac{b}{a}\right)^{v t}-1}\right) \\
& \times\left(\frac{a^{-v t}(v t)^{k}}{1-y\left(\lambda\left(\frac{b}{a}\right)^{v t}-1\right)}\right)^{m} c^{u v z t}\left(\frac{\left(\frac{\lambda y}{y+1}\right)^{v}\left(\frac{b}{a}\right)^{u v t}-1}{\frac{\lambda y}{y+1}\left(\frac{b}{a}\right)^{u t}-1}\right) \cdot \\
= & \frac{(y+1)^{u-1}(y+1)^{v-1}}{(u v)^{k m}} \sum_{i=0}^{u-1} \sum_{j=0}^{v-1}\left(\frac{\lambda y}{y+1}\right)^{i+j}\left(\frac{b}{a}\right)^{(i v+j u) t} c^{v x(u t)}\left(\frac{a^{-u t}(u t)^{k}}{1-y\left(\lambda\left(\frac{b}{a}\right)^{u t}-1\right)}\right)^{m}
\end{aligned}
$$




$$
\begin{aligned}
& \times\left(\frac{a^{-v t}(v t)^{k}}{1-y\left(\lambda\left(\frac{b}{a}\right)^{v t}-1\right)}\right)^{m} c^{u z(v t)} \\
= & \frac{(y+1)^{u-1}(y+1)^{v-1}}{(u v)^{k m}}\left[\sum_{i=0}^{u-1} \sum_{j=0}^{v-1}\left(\frac{\lambda y}{y+1}\right)^{i+j} \sum_{n=0}^{\infty} \mathcal{F}_{n, k}^{(m)}\left(v x+\left(i \frac{v}{u}+j\right) \log _{c}(b / a), y ; a, b, c ; \lambda\right) \frac{(u t)^{n}}{n !}\right] \\
& \times \sum_{n=0}^{\infty} \mathcal{F}_{n, k}^{(m)}(u z, y ; a, b, c ; \lambda) \frac{(v t)^{n}}{n !} \\
= & \frac{(y+1)^{u+v-2}}{(u v)^{k m}} \sum_{n=0}^{\infty}\left[\sum_{r=0}^{n}\left(\begin{array}{l}
n \\
r
\end{array}\right) \sum_{i=0}^{u-1} \sum_{j=0}^{v-1}\left(\frac{\lambda y}{y+1}\right)^{i+j} u^{r} v^{n-r} \mathcal{F}_{r, k}^{(m)}\left(v x+\left(i \frac{v}{u}+j\right) \log _{c}(b / a), y ; a, b, c ; \lambda\right)\right. \\
\times & \left.\mathcal{F}_{n-r, k}^{(m)}(u z, y ; a, b, c ; \lambda)\right] \frac{t^{n}}{n !} .
\end{aligned}
$$

Similarly,

$$
\begin{aligned}
L(t)= & \frac{(y+1)^{u-1}(y+1)^{v-1}}{(u v)^{k m}}\left(\frac{a^{-v t}(v t)^{k}}{1-y\left(\lambda\left(\frac{b}{a}\right)^{v t}-1\right)}\right)^{m} c^{u x(v t)}\left(\frac{\left(\frac{\lambda y}{y+1}\right)^{v}\left(\frac{b}{a}\right)^{u v t}-1}{\frac{\lambda y}{y+1}\left(\frac{b}{a}\right)^{u t}-1}\right) \\
& \times\left(\frac{\left(\frac{\lambda y}{y+1}\right)^{u}\left(\frac{b}{a}\right)^{u v t}-1}{\frac{\lambda y}{y+1}\left(\frac{b}{a}\right)^{v t}-1}\right)\left(\frac{a^{-u t}(u t)^{k}}{1-y\left(\lambda\left(\frac{b}{a}\right)^{v t}-1\right)}\right)^{m} c^{v z(u t)} . \\
= & \frac{(y+1)^{u+v-2}}{(u v)^{k m}} \sum_{n=0}^{\infty}\left[\sum_{r=0}^{n}\left(\begin{array}{l}
n \\
r
\end{array}\right) \sum_{i=0}^{v-1} \sum_{j=0}^{u-1}\left(\frac{\lambda y}{y+1}\right)^{i+j} v^{r} u^{n-r} \mathcal{F}_{r, k}^{(m)}\left(u x+\left(i \frac{u}{v}+j\right) \log _{c}(b / a), y ; a, b, c ; \lambda\right)\right. \\
& \left.\times \mathcal{F}_{n-r, k}^{(m)}(v z, y ; a, b, c ; \lambda)\right] \frac{t^{n}}{n !} .
\end{aligned}
$$

Combining (16) and (17) gives the desired identity.

Setting $y=-\frac{1}{2}$ and $k=0$ in Theorem 7 , we obtain the following corollary.

Corollary 18. For $u, v, m \in \mathbb{N}$ and $n \in \mathbb{N}_{0}$, we have

$$
\begin{aligned}
& \sum_{r=0}^{n}\left(\begin{array}{l}
n \\
r
\end{array}\right) \sum_{i=0}^{u-1} \sum_{j=0}^{v-1}(-\lambda)^{i+j} u^{r} v^{n-r} E_{r}^{(m)}\left(v x+\left(i \frac{v}{u}+j\right) \log _{c}(b / a) ; a, b, c ; \lambda\right) E_{n-r}^{(m)}(u z ; a, b, c ; \lambda) \\
& =\sum_{r=0}^{n}\left(\begin{array}{l}
n \\
r
\end{array}\right) \sum_{i=0}^{v-1} \sum_{j=0}^{u-1}(-\lambda)^{i+j} v^{r} u^{n-r} E_{r}^{(m)}\left(u x+\left(i \frac{u}{v}+j\right) \log _{c}(b / a) ; a, b, c ; \lambda\right) E_{n-r}^{(m)}(v z ; a, b, c ; \lambda) .
\end{aligned}
$$

Setting $y=-2, k=1$ and replacing $\lambda$ by $\frac{\lambda}{2}$ in Theorem 7, we obtain the following corollary. 
Corollary 19. For $u, v, m \in \mathbb{N}$ and $n \in \mathbb{N}_{0}$, we have

$$
\begin{aligned}
& \sum_{r=0}^{n}\left(\begin{array}{l}
n \\
r
\end{array}\right) \sum_{i=0}^{u-1} \sum_{j=0}^{v-1}(\lambda)^{i+j} u^{r} v^{n-r} B_{r}^{(m)}\left(v x+\left(i \frac{v}{u}+j\right) \log _{c}(b / a) ; a, b, c ; \lambda\right) B_{n-r}^{(m)}(u z ; a, b, c ; \lambda) \\
& =\sum_{r=0}^{n}\left(\begin{array}{l}
n \\
r
\end{array}\right) \sum_{i=0}^{v-1} \sum_{j=0}^{u-1}(\lambda)^{i+j} v^{r} u^{n-r} B_{r}^{(m)}\left(u x+\left(i \frac{u}{v}+j\right) \log _{c}(b / a) ; a, b, c ; \lambda\right) B_{n-r}^{(m)}(v z ; a, b, c ; \lambda) .
\end{aligned}
$$

Setting $y=-\frac{1}{2}$ and $k=1$ in Theorem 7 , we obtain the following corollary.

Corollary 20. For $u, v, m \in \mathbb{N}$ and $n \in \mathbb{N}_{0}$, we have

$$
\begin{aligned}
& \sum_{r=0}^{n}\left(\begin{array}{l}
n \\
r
\end{array}\right) \sum_{i=0}^{u-1} \sum_{j=0}^{v-1}(-\lambda)^{i+j} u^{r} v^{n-r} G_{r}^{(m)}\left(v x+\left(i \frac{v}{u}+j\right) \log _{c}(b / a) ; a, b, c ; \lambda\right) G_{n-r}^{(m)}(u z ; a, b, c ; \lambda) \\
& =\sum_{r=0}^{n}\left(\begin{array}{l}
n \\
r
\end{array}\right) \sum_{i=0}^{v-1} \sum_{j=0}^{u-1}(-\lambda)^{i+j} v^{r} u^{n-r} G_{r}^{(m)}\left(u x+\left(i \frac{u}{v}+j\right) \log _{c}(b / a) ; a, b, c ; \lambda\right) G_{n-r}^{(m)}(v z ; a, b, c ; \lambda) .
\end{aligned}
$$

Setting $a=1$ and $b=c=e$ in Theorem 7 , we obtain another symmetry identity for the polynomials $F_{n, k}^{(\alpha)}(x, y ; \lambda)$.

Corollary 21. For $u, v, m \in \mathbb{N}, n \in \mathbb{N}_{0}$ and $y \neq-1$, we have

$$
\begin{aligned}
& \sum_{r=0}^{n}\left(\begin{array}{l}
n \\
r
\end{array}\right) \sum_{i=0}^{u-1} \sum_{j=0}^{v-1}\left(\frac{\lambda y}{y+1}\right)^{i+j} u^{r} v^{n-r} F_{r, k}^{(m)}\left(v x+i \frac{v}{u}+j, y ; \lambda\right) F_{n-r, k}^{(m)}(u z, y ; \lambda) \\
& =\sum_{r=0}^{n}\left(\begin{array}{l}
n \\
r
\end{array}\right) \sum_{i=0}^{v-1} \sum_{j=0}^{u-1}\left(\frac{\lambda y}{y+1}\right)^{i+j} v^{r} u^{n-r} F_{r, k}^{(m)}\left(u x+i \frac{u}{v}+j, y ; \lambda\right) \mathcal{F}_{n-r, k}^{(m)}(v z, y ; \lambda) .
\end{aligned}
$$

Taking $y=-\left(2^{k-1} a^{b}+1\right)$ and $\lambda=\frac{2^{k-1} \beta^{b}}{2^{k-1}+1}$ in Corollary 21, we get Theorem 3.9 of [25].

Corollary 22. For $a, b>0 ; \beta \in C ; u, v, m \in \mathbb{N}$ and $n \in \mathbb{N}_{0}$, we have

$$
\begin{aligned}
& \sum_{r=0}^{n}\left(\begin{array}{l}
n \\
r
\end{array}\right) \sum_{i=0}^{u-1} \sum_{j=0}^{v-1}\left(\frac{\beta}{a}\right)^{b(i+j)} u^{r} v^{n-r} P_{r, \beta}^{(m)}\left(v x+i \frac{v}{u}+j ; k, a, b\right) P_{n-r, \beta}^{(m)}(u z ; k, a, b) \\
& =\sum_{r=0}^{n}\left(\begin{array}{l}
n \\
r
\end{array}\right) \sum_{i=0}^{v-1} \sum_{j=0}^{u-1}\left(\frac{\beta}{a}\right)^{b(i+j)} v^{r} u^{n-r} P_{r, \beta}^{(m)}\left(u x+i \frac{u}{v}+j ; k, a, b\right) P_{n-r, k}^{(m)}(v z ; k, a, b) .
\end{aligned}
$$

\section{Acknowledgements}

The author greatly appreciates the anonymous reviewers for their valuable comments and suggestions for this paper. 


\section{References}

[1] S. Araci, W.A. Khan, M. Acikgoz, C. Ozel, and P. Kumam. A new generalization of Apostol-type Hermite-Genocchi polynomials and its application. Springerplus, 5(860):17 pages, 2016.

[2] K.N. Boyadzhiev. A series transformation formula and related polynomials. Int. J. Math. Sci., 23:3849-3866, 2005.

[3] R. Dere and Y. Simsek. Hermite base Bernoulli type polynomials on the umbral algebra. Russian Journal of Mathematical Physics, 22(1):1-5, 2015.

[4] R. Dere, Y. Simsek, and H.M. Srivastava. A unified presentation of three families of generalized Apostol type polynomials based upon the theory of the umbral calculus and the umbral algebra. J. Number Theory, 133:3245-3263, 2013.

[5] B.N. Guo and F. Qi. Generalization of Bernoulli polynomials. J. Math. Ed. Sci. Tech., 33(3):428-31, 2002.

[6] G.-W. Jang and T. Kim. Some identities of ordered Bell numbers arising from differential equations. Adv. Stud. Contemp. Math(Kyungshang), 27(3):385-397, 2017.

[7] H. Jolany and R. Corcino. Explicit formula for the Generalization of poly-Bernoulli numbers and polynomials with $a, b, c$ parameters. J. Class. Anal., 6(2):119-135, 2015.

[8] H. Jolany, H. Sharifi, and R. Alikelaye. Some results for the Apostol-Genocchi polynomials of higher order. The Bulletin of Malaysia Soc., 2:465-479, 2013.

[9] B.K. Karande and N.K. Thakare. On the unification of Bernoulli and Euler polynomials. Indian J. Pure Appl. Math, 6:98-107, 1975.

[10] L. Kargin. Some formulae for products of geometric polynomials with applications. Journal of Integer Sequences, 20(Article 17.4.4):15 pages, 2017.

[11] N. Kilar and Y. Simsek. A new family of Fubini type numbers and polynomials associated with Apostol-Bernoulli numbers and polynomials. J. Korean Math. Soc., 54:1605-1621, 2017.

[12] D.S. Kim, G.-W. Jang, H.-I. Kwon, and T. Kim. Two variable higher-order degenerate Fubini polynomials. Proc. Jangjeon Math. Soc., 21(1):5-22, 2018.

[13] D.S. Kim, T. Kim, H.-I. Kwon, and J.-W. Park. Two variable higher order Fubini polynomials. J. Korean Math. Soc., 55(4):975-986, 2018.

[14] T. Kim. Degenerate ordered Bell numbers and polynomials. Proc. Jangjeon Math Soc., 20(2):137-144, 2017.

[15] T. Kim, D.S. Kim, G. Jang, and D. Kim. Two variable higher-order central Fubini polynomials. J. Inequal. Appl, 146, 2019. 
[16] T. Kim, D.S. Kim, and G.-W. Jang. A note on degenerate Fubini polynomials. Proc. Jangjeon Math. Soc., 20:521-531, 2017.

[17] T. Kim, D.S. Kim, G.-W. Jang, and J. Kwon. Symmetric identities for Fubini polynomials. Symmetry, 10(6):219, 7 pages, 2018.

[18] V. Kurt. A further symmetric relation on the analogue of the Apostol-Bernoulli and the analogue of the Apostol-Genocchi polynomials. Appl. Math. Sci., 3(56):27572764, 2009.

[19] D.Q. Lu and H.M. Srivastava. Some series identities involving the generalized Apostol type and related polynomials. Comp. Math. Appl., 62(2011):3591-3602.

[20] Q.-M. Luo. On the Apostol-Bernoulli polynomials. Central Eur. J. Math., 2:509-515, 2004 .

[21] Q.-M. Luo. Apostol-Euler polynomials of higher order and Gaussian hypergeometric functions. Taiwanese J Math., 10(4):917-925, June 2006.

[22] Q.-M. Luo. Fourier expansions and integral representations for the Genocchi polynomials. J. Integer Seq., 12(Article 09.1.4), 2009.

[23] Q.-M. Luo. Extension for the Genocchi polynomials and its Fourier expansions and integral representations. Osaka J. Math., 48(2):291-309, 2011.

[24] Q.-M. Luo and H.M. Srivastava. Some generalizations of the Apostol-Bernoulli and Apostol-Euler polynomials. J. Math. Anal. Appl., 308(1):290-302, 2005.

[25] M.A. Ozarslan. Unified Apostol-Bernoulli, Euler and Genocchi polynomials. Comput. Math. Appl., 62(6):2452-2462, 2011.

[26] H. Ozden, Y. Simsek, and H.M. Srivastava. A unified presentation of the generating functions of the generalized Bernoulli, Euler and Genocchi polynomials. Comput. Math Appl, 60(10):2779-2787, 2010.

[27] S.-S. Pyo. Some identities of degenerate Fubini polynomials arising from differential equations. J. Nonlinear Sci. Appl., 11(3):383-393, 2018.

[28] H. M. Srivastava, B. Kurt, and Y. Simsek. Some families of Genocchi type polynomials and their interpolation functions. Integral Transform and Special Functions, 23(12):919-938, 2012. DOI:10.1080/10652469.2011.643627.

[29] H.M. Srivastava and J. Choi. Series Associated with the Zeta and Related Functions. Kluwer Academic, Dordrecht, 2001.

[30] H.M. Srivastava, M. Garg, and S. Choudhary. A new generalization of the Bernoulli and Related Polynomials. Russian J. Math. Physics, 17(2):251-261, 2010. 
[31] H.M. Srivastava, M. Garg, and S. Choudhary. Some new families of generalized Euler and Genocchi polynomials. Taiwanese J. Math., 15(1):283-305, 2011.

[32] H.M. Srivastava and P.G. Todorov. An explicit formula of the generalized Bernoulli polynomials. J Math Anal. Appl., 130:509-513, 1988.

[33] Z. Zhang and H. Yang. Several identities for the generalized Apostol-Bernoulli polynomials. Comput. Math. Appl., 56(12):2993-2999, 2008. 